\title{
A study of the transient processes in the physical model of the electric power systems with predominant part of the renewable energy sources
}

\author{
Ivaylo Nedelchev ${ }^{1 *}$, Hristo Zhivomirov ${ }^{1}$, and Yoncho Kamenov ${ }^{2}$ \\ ${ }^{1}$ Technical University of Varna, Department of Theory of Electrical Engineering and Measurements, \\ Bulgaria \\ ${ }^{2}$ Technical University of Varna, Department of Power Engineering, Bulgaria
}

\begin{abstract}
The renewable energy take part in the most of the electric power systems in the modern world. The part of this type of energy in the global electric power system, as well as in the local scale, increases with the setting the stricter requirements for decreasing the level of the carbon dioxide emissions. This is the result of the newest international conventions and decision for saving the nature. By these conditions, the electric power systems are forced to work with more different types of energy sources: wind power, photovoltaic, biomass plants etc. Switching of such miscellaneous power sources, leads to complicated transient processes, which are developed due to specific electrical parameters, especially harmonic components, of the synchronous generators, photovoltaic and wind power plants. This paper represents data from measurements of the transient processes into the physical model of the electric power system with predominant part of renewable energy and assesses the applicability of the model. For conducting this study, the multichannel DAQ measurement system is used.
\end{abstract}

\section{Introduction}

During the last few decades, the scale of renewable energy sources (RES) integrated into the electric power system (EPS) has been rapidly increasing. In the global EPS, there is a real possibility to appear a case with a predominant part of the renewable energy in a large region or during a long time period $[1,2,3]$. In the global EPS there exist the regions with predominant part of RES [2]. In those regions, one or more types of RES could exist. The most often cases of RES types are: wind power turbines and photovoltaic modules [4]. All of RES electrical schemes incorporate inverter, with switch-mode power supply (SMPS) module, which creates a high level of high frequency powerful harmonics, decreasing the power factor in the circuit, quality of the power energy, sustainability and reliability of the system $[1,4,5]$. These effects increase the risk of unpredictable and

\footnotetext{
* Corresponding author: iynedelchev@abv.bg
} 
unprovoked failures and collapse of the system. That type of effect was the reason for the failure of the system in large regions in Western Europe in the beginning of 2021.

This paper represents a study of the processes in a small-scale physical model of the EPS with predominant part of the RES $[6,7,8]$. The purpose of this study is to represent the verification and applicability of the constructed physical model, as well as the possibility for monitoring of the transient processes during system mode adjusting, changing of the level of the load of the power lines, and quality of the generated electric energy from different types of RES.

\section{Short description and connecting diagram of the physical model}

In every EPS, there exist different types power units of RES. The most used are: wind power turbines, photovoltaic plant, water turbine generators, biomass power plants. The constructed physical model is designed from the team of the power-engineering department in the Technical university of Varna [6]. For the simulation of the wind power and sun radiation power, the real inverters with DC voltage source are used. Simulating the RES is performed via adjustable DC voltage source (ADCV). With the help of controllable threephase power rectifiers the inverters was sourced with DC voltage at the input side. Thus, the energy regime of this type of power units can be changed via adjusting the mode of the rectifiers. In the model, one of the inverters represents a photovoltaic generator (PVG) and the other one - wind power generator (WPG). For representing of the water turbine generator in the system, the direct coupled motor synchronous generators are included (SG1, SG2, SG3). Multi-stage resistive loads are used as consumers of the lines.

On the Fig. 1 is shown the diagram of the physical model. The main studied power units in the diagram are marked as: synchronous generators (SG1, SG2, SG3), photovoltaic generator (PVG) and Wind power generator (WPG). The terminals of the resistive loads are marked as L1 - L7.

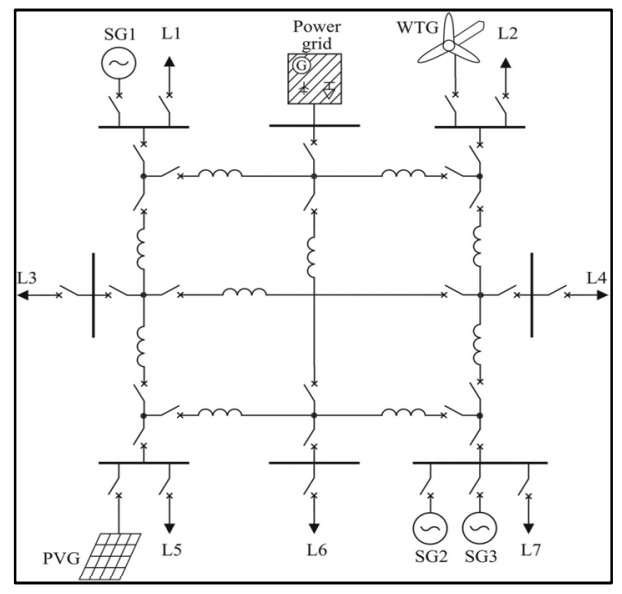

Fig. 1. Single line diagram of the physical model as an object of the measurement ( $c f$ Fig.1 in [6]).

The inductors in the diagram represent the inductance of the transmission lines. The synchronization of the SG1-3 is implemented by controllers and all requirements for the synchronization to the global power net, concerning voltage frequency, phase angle, phase sequence and waveform of the generated voltage are fulfilled. Two types of controllers are used: for internal synchronization between the different units in the model structure and for 
external synchronization during the connection to the global power net. The model is capable for working in so-called "island mode". In this mode, the synchronization of the SG 1-3 can be done manually or automatically. Detailed diagram of the power units and description of the physical model are given in [6].

\section{Measurement equipment. Connecting diagrams, transducers and software}

The experiments was conducted via measurement set, which consist of: DAQ module NI-6211, supplement primary transducers and software. The connecting diagram for the cases of measurements including RES power units or synchronous generators are given on the Fig.2 and Fig.3.

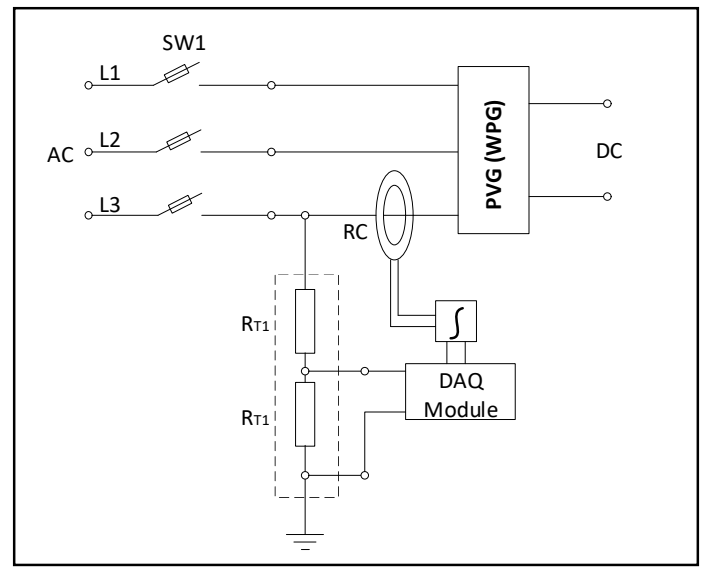

Fig. 2. Measurement the current and voltage in the output circuit of the simulated photovoltaic generator $(\mathrm{PVG})$ or wind power generator (WPG).

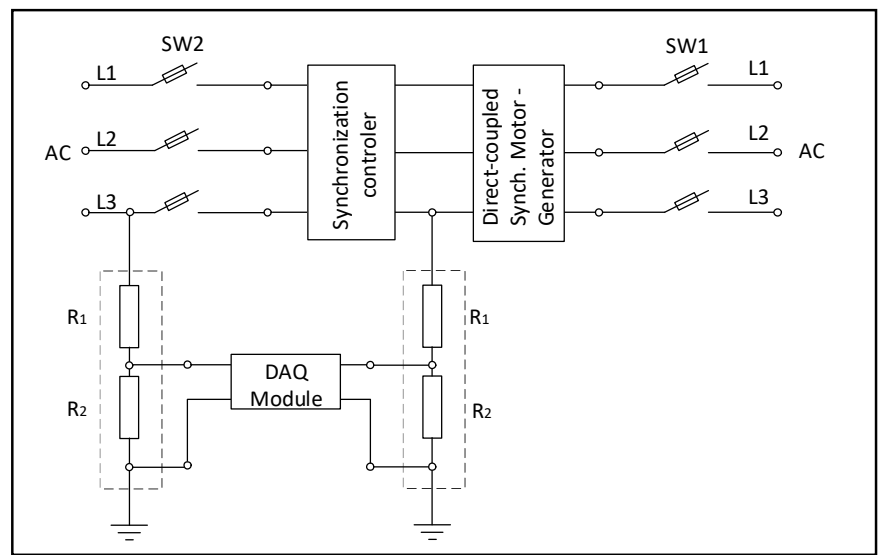

Fig. 3. Measurement of the input and output voltage in the circuit of the synchronous generator (SG).

Due to the symmetric type of the load, it was measured the output voltage and current in a single phase of the three-phase type of EPS. In other cases, the input or output voltages of the single power unit was observed. In this way, for measurements two of the channels of the DAQ module was involved. For the measurement of the voltage it was used a highvoltage divider with a large input resistance [9]. As a current transducer, the Rogowski coil 
with incorporated integrator was used [10]. For the purpose of the experiment, the LabVIEW software was involved and as a result, was obtained the time and frequency domain graphs.

The main parameters and conditions of the experimental set are:

- Sampling rate of the DAQ system - $5000 \mathrm{~Hz}$;

- Input resistance of the high-voltage divider - $1 \mathrm{G} \Omega$;

- Frequency band of the Rogowski coil measurement module $-100 \mathrm{kHz}$;

- Current range of the Rogowski coil - 100 A;

- Load of the power units - the load was gradually changed through three levels: $10 \%$, $50 \%, 100 \%$; For measuring of the biggest effect of the transient process, a step changing of the load was used from $10 \%$ to $100 \%$;

- The resistive load - the rate of the involvong the resistive load in the energy transmission lines was executed via IGBT switch regulator. The levels of the resistive load were adjusted from $10 \%$ of the nominal load with the step of $10 \%$ up to the nominal capacity of the load. - Coefficient of transformation for the Rogowski coil is [10]:

$$
K_{R C}=\frac{I_{1}}{U_{2}}=2000 \frac{A}{V^{\prime}}
$$

and for the high-voltage divider is:

$$
K_{d}=\frac{U_{2}}{U_{1}}=2000
$$

On the Fig.4 is shown the WPG power unit box of the physical model and DAQ system during the measurements [6].

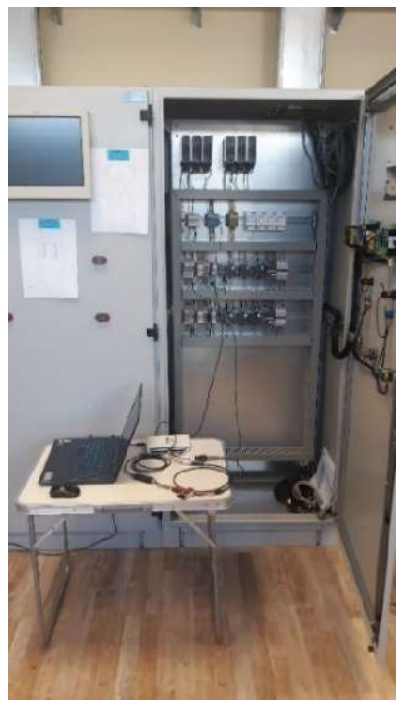

Fig. 4. Common view of the part of the physical model (WPG power unit) and measurement equipment.

\section{Parameters of the transient process of the studied physical RES model}

During the operation of the RES into the EPS system, the energy generation passes through three stages [11]:

- synchronization, of the power units with the main EPS, 
- establishment of the frequency and amplitude - energy transfer into the EPS;

- disconnecting the power units from the EPS.

It could be distinguished one more stage case - the changing of the operation mode of the RES.

Usually switching on the RES and disconnecting from the EPS requires a few seconds in order to set stable parameters of generation [11]. From the other hand, the quality of the generated energy also is important factor for system stability. For example, the output voltage shape of the inverters and noise in the system are one of the strongest factors for system failures [1]. Having in mind upper reasons and facts it will be correct the parameters of the model transient process to be defined as follows [11]:

- time for synchronisation with the EPS frequency $-\boldsymbol{t}_{\boldsymbol{s}}, \boldsymbol{s}$.

$\boldsymbol{t}_{\boldsymbol{s}}$ is the time between switching on the power units into the EPS and establishment of the frequency and in-phase mode of the module in the system;

- time for disconnecting - $\boldsymbol{t}_{\boldsymbol{d}}, \boldsymbol{s}$.

$\boldsymbol{t}_{\boldsymbol{d}}$ is the time between switching off the power units from the EPS and attenuation of their output voltage to the $\boldsymbol{U}_{\boldsymbol{m}} / \boldsymbol{e}$ level, where $\boldsymbol{U}_{\boldsymbol{m}}$ is the amplitude of the nominal output voltage before involving the system onto the transient mode.

- time for switching the mode $-\boldsymbol{t}_{\boldsymbol{s m}}, \boldsymbol{s}$.

$\boldsymbol{t}_{\boldsymbol{s} \boldsymbol{m}}$ is the time for switching of the power between two different modes. Considering the stable levels of operation, the largest difference in working modes are by load level between $10 \%$ to $100 \%$ of the nominal regime.

The parameters $\boldsymbol{t}_{\boldsymbol{d}}, \boldsymbol{t}_{\boldsymbol{s}}$ are most applicable for monitoring the output voltage of the synchronous generators (SG1, SG2, SG3), while the parameter $\boldsymbol{t}_{\boldsymbol{s} m}$, is more useful for PVG and WPG. All of them are important transient mode parameters, which characterize flexibility of the EPS with respect to outer RES impact.

- total harmonic distortion of the power units - $\boldsymbol{T H D}$;

The expression for THD is given by:

$$
T H D=\frac{\sqrt{\sum_{n=2}^{N} U_{n}^{2}}}{U_{1}},
$$

where $\boldsymbol{n}$ is the highest harmonic of the measurement set, $\boldsymbol{U}_{\boldsymbol{I}}$ is the RMS value of the fundamental harmonic voltage in the system, $\boldsymbol{U}_{\boldsymbol{n}}$ are the voltage harmonics with the numbers higher than fundamental one (RMS value).

- the impact of the higher harmonic (percentage from the basic one) $-\boldsymbol{\delta} \boldsymbol{U}_{\boldsymbol{n}}, \%\left(\boldsymbol{U}_{\boldsymbol{n}}-\right.$ the biggest RMS voltage value from the sequence of the higher harmonics of the RES or synchronous generator; $\boldsymbol{n}$ is the number of the certain harmonic)

$$
\delta U=\frac{U_{n}}{U_{1}} \cdot 100, \%
$$

\section{Measurement results}

\subsection{Parameters of the transient process}

The measured parameters of the transient process, which characterize the behaviour of the power units and resistive load regulator in the physical model, are given in the Table 1. The quality of the generated energy depends on the level of the THD and $\delta U_{n}$. They are obtained by $50 \%$ of the nominal load level of the system. The biggest distortion were obtained by SG power unit $-10,97 \%$ and the lowest level by PVG $-2,54 \%$. In the Table 1 , $n$ is the number of the largest higher harmonic. The other frequency components also have 
the similar RMS level such, as the measured biggest one higher harmonic. The impact of the higher harmonics is assessed with the THD parameter.

Table 1. Observed parameters of the transient process of the studied power units and resistive load.

\begin{tabular}{|c|c|c|c|c|}
\hline & SG & PVG & WPG & LA \\
\hline THD $\%$ & 0,83 & 0,91 & 1,95 & 23,55 \\
\hline$\delta U_{n}, \%$ & 10,97 & 7,73 & 2,54 & 35,9 \\
\hline$n$ & 5 & 5 & 7 & 3 \\
\hline
\end{tabular}

Via spectral analysis of the output power unit voltages, their higher harmonics level is obtained. In the Table 2 are represented further calculations of the times of the transient process for synchronization and disconnecting $\left(t_{s}, t_{d}\right)$ of the SG1 from the circuit. Depending on the defined power units also, the switching mode times $t_{s m}$ of input (DC) voltages for the both inverters representing PVG and WPG are found to be $0,5 \mathrm{~s}$. The AC switching times mode of the inverters' voltages have approximately the same level - 0,54 $0,55 \mathrm{~s}$. During the synchronization with the grid and disconnecting the SG1, this module shows different behavior. The time for synchronization $t_{s}$, is approximately three times more than disconnecting time $t_{d}$.

Table 2. Synchronization periods of power units by switching between different modes.

\begin{tabular}{|c|c|c|c|c|c|}
\hline Synchronization periods & SG & $\begin{array}{c}\text { PVG } \\
(\mathrm{AC})\end{array}$ & $\begin{array}{c}\text { PVG } \\
(\mathrm{DC})\end{array}$ & $\begin{array}{c}\text { WPG } \\
(\mathrm{AC})\end{array}$ & $\begin{array}{c}\text { WPG } \\
\text { (DC) }\end{array}$ \\
\hline $\begin{array}{c}t_{s}, s \\
\text { (by load level of } 50 \%)\end{array}$ & 7,7 & - & - & - & - \\
\hline $\begin{array}{c}t_{d}, s \\
\text { (by load level of } 50 \%)\end{array}$ & 2,9 & - & - & - & - \\
\hline $\begin{array}{c}t_{s m}, s \\
\text { (by swithching from } \\
10 \% \text { to } 100 \% \text { of the load level) }\end{array}$ & - & 0,55 & 0,5 & 0,54 & 0,5 \\
\hline
\end{tabular}

\subsection{Time domain graphs of the measured transient process}

The most detailed information of the studied transient processes in the physical model, can be represented via the graphs across the time domain and frequency domain. Fig.5 Fig. 7 show the time domain graphs for the SG1 power unit.

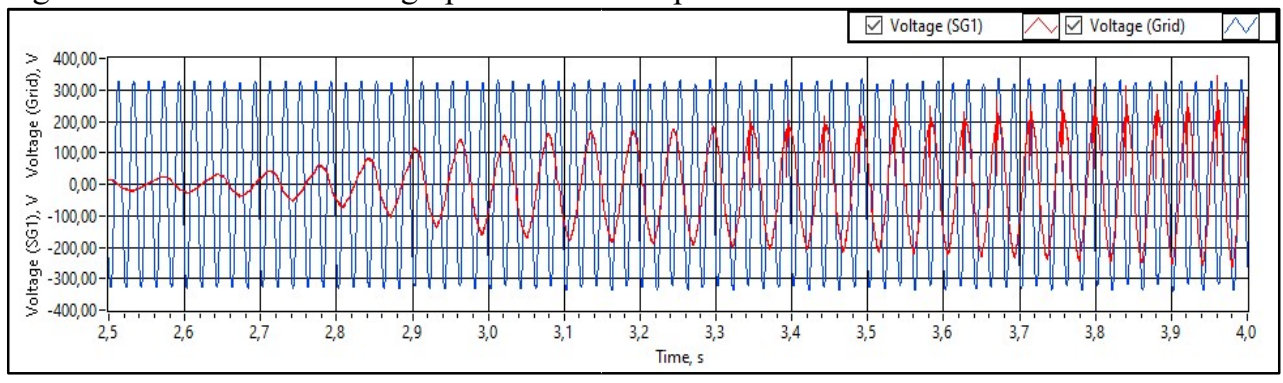

Fig. 5. The process of the switching the SG1 power unit into the grid.

On the time domain graphs, the defined transient process times, shown in the Table 2 can be measured. The synchronization of the SG module occurs, when synchronization controller (Fig.3), detect matching in phase the both voltages - the output of the SG1 and the grid voltage. Then the switch SW2 is closing. It can be mentioned, the beginning of the long period for synchronization (more than $7 \mathrm{~s}$.), which is calculated from the software but 
prolongs after the diagram on the Fig. 5. Unlike, by disconnecting, the level of the output voltage fades away within $2,5 \mathrm{~s}$. pseudo periodically.

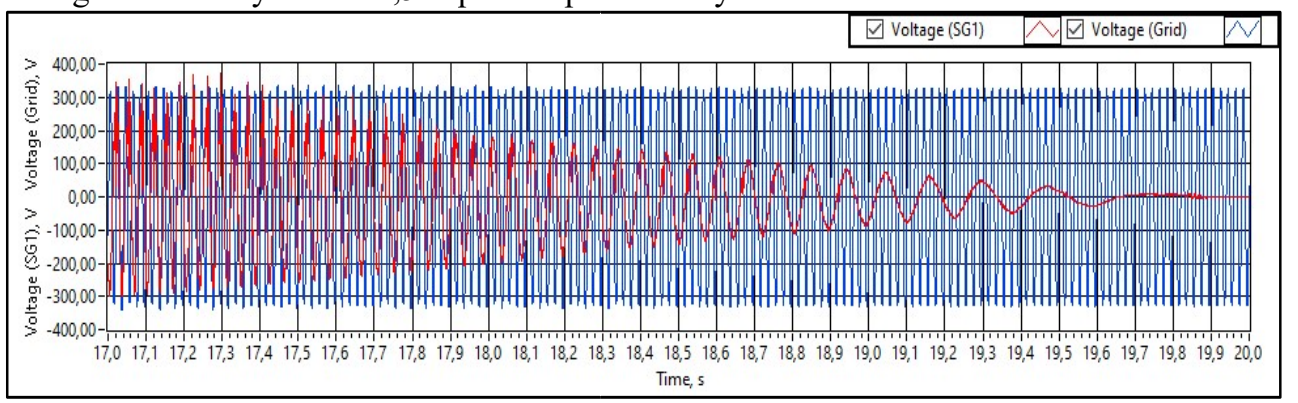

Fig. 6. The process of disconnecting the SG1 from the grid (common view).

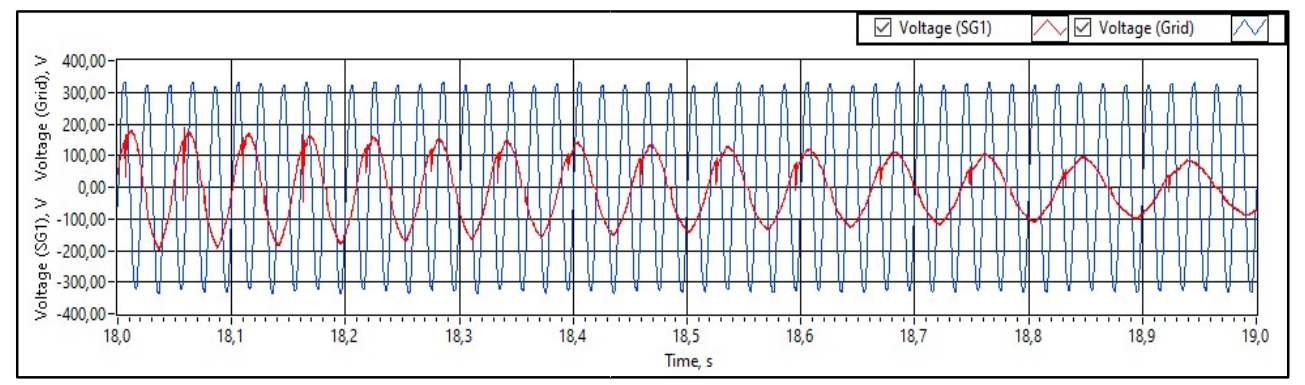

Fig. 7. The process of disconnecting the SG1 from the grid (larger scale). Fading the oscillations of the SG1.

The process of the changing the load level from $10 \%$ to $100 \%$ of the nominal mode for the AC and DC side of the WPG unit is represented on the Fig.8 and Fig.9.

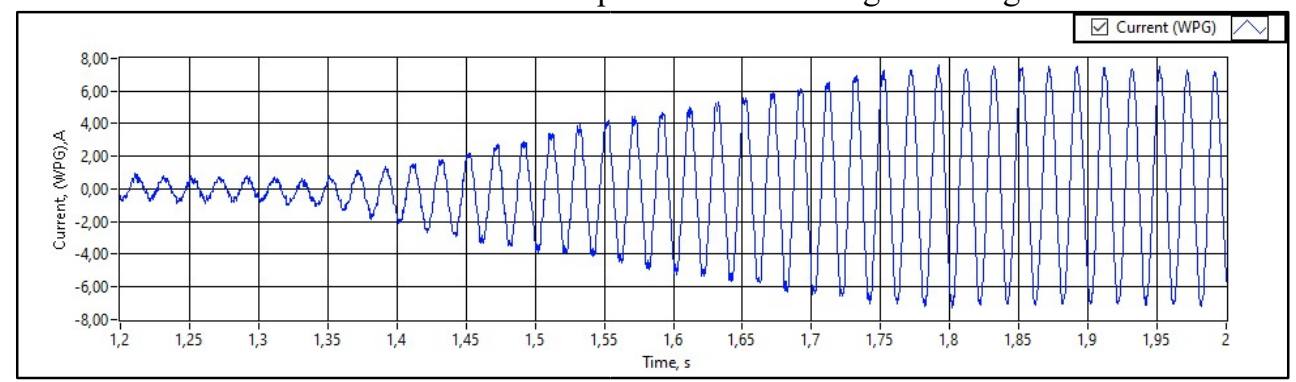

Fig. 8. The process of switching the WPG from $10 \%$ to $100 \%$ of the load (AC current).

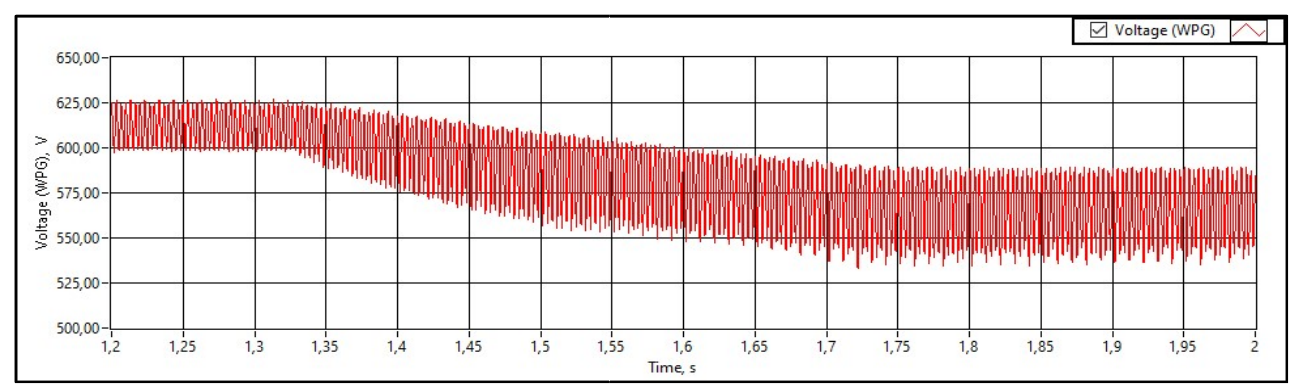

Fig. 9. The process of switching the WPG from $10 \%$ to $100 \%$ of the load (DC voltage). 
Sharply increasing of the current load, can be seen on the Fig.8. It can be observed establishing the level of the raised current of the AC side of the WPG unit and on the other hand, the decreasing of the source voltage in the DC part of the inverter (Fig. 9). The different period times of the transient process can be distinguished easily.

Another dynamic process in the model can be observed during the changing the level of the load of the power line. Fig. 10 and Fig. 11 show graphs, which represent the operation of the current output circuit in the IGBT regulator and its frequency response. It can be mentioned the rich spectral set and the big level of the higher harmonics. The spectral analysis shows a high level of distortion. THD of the output current exceed $23 \%$.

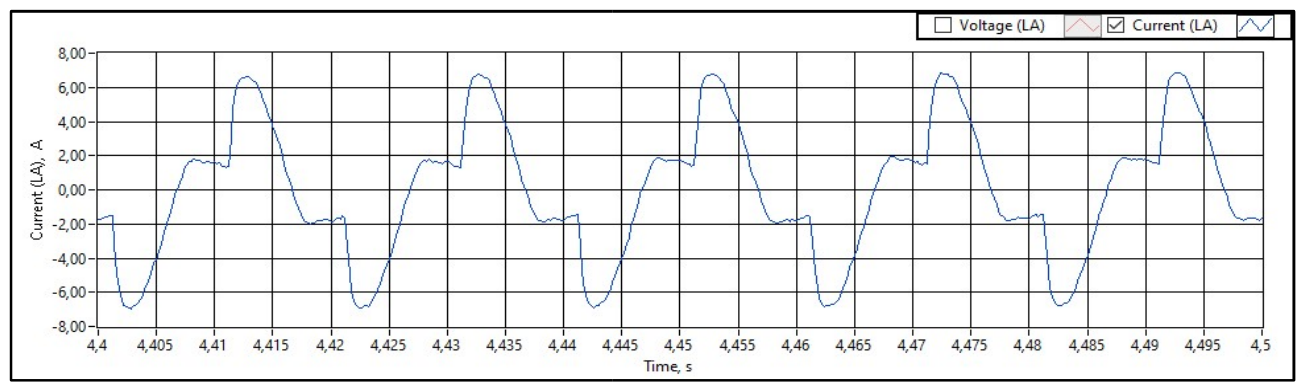

Fig. 10. The time domain of the current by $70 \%$ level of the resistive load, regulated by IGBT switch.

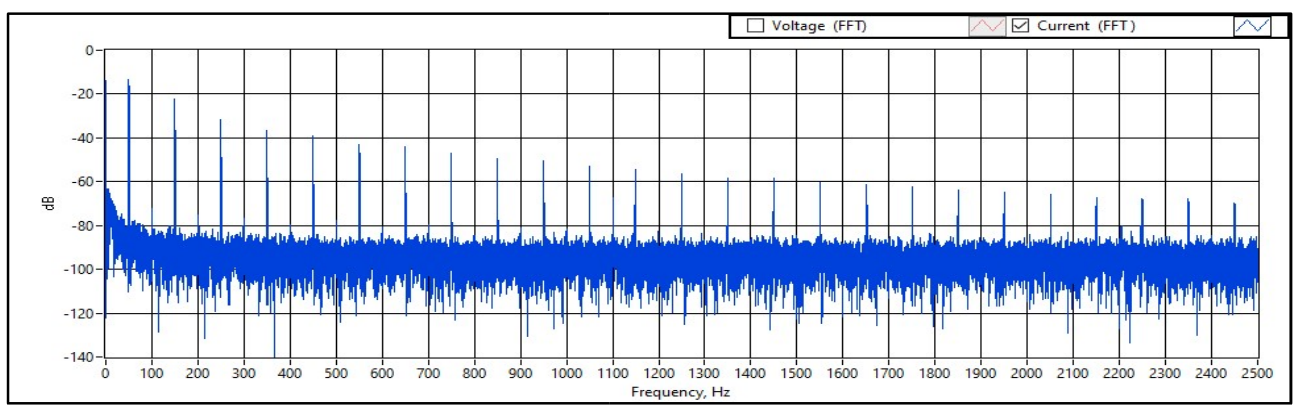

Fig. 11. The frequency domain of the current by $70 \%$ level of the resistive load, regulated by IGBT switch.

The moment of switching between different modes of the active loads or initially turning the power generation on, are shown in the Fig.12. The drift of the output current is obvious and its appearance is due to the saved energy in the parasitic elements in the output circuit. Both capacitance of the switching elements and stray inductance parameters of the power line generate a pseudo periodic process of the output current circuit.

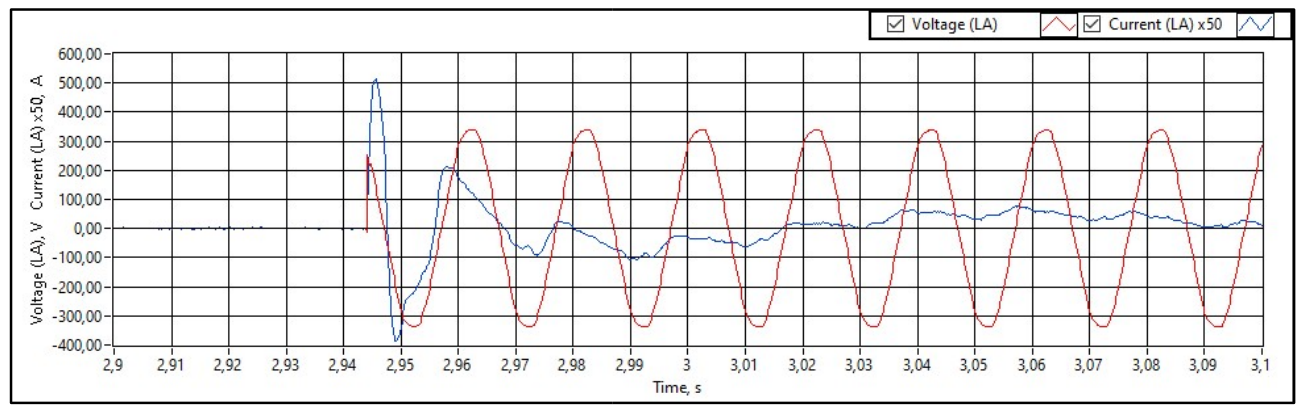

Fig. 12. The time domain graph of the current and voltage by the switching moment in the power line. 


\subsection{Frequency domain graphs of the measured transient process}

In the studying physical model of RES, about the considering power generating units, as it is known, the rich composition of frequency components have to be present. On the frequency domain graphs are shown the spectral sequences of the higher level harmonics, which modulate the output quantities of the power units. The predominant part of them, consists of odd harmonics. Fig. 13 - Fig. 15 represents normalized frequency domain graphs of the output SG1 voltage, WPG and PVG currents (the biggest impact of the higher harmonics are enclosed on the graph area).

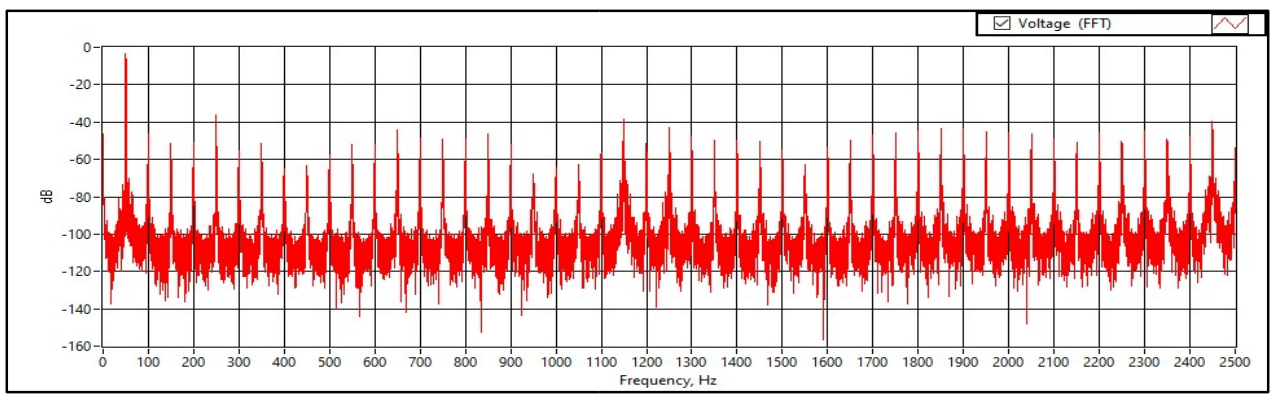

Fig. 13. The spectral analysis of the SG1 voltage, synchronized with the grid voltage.

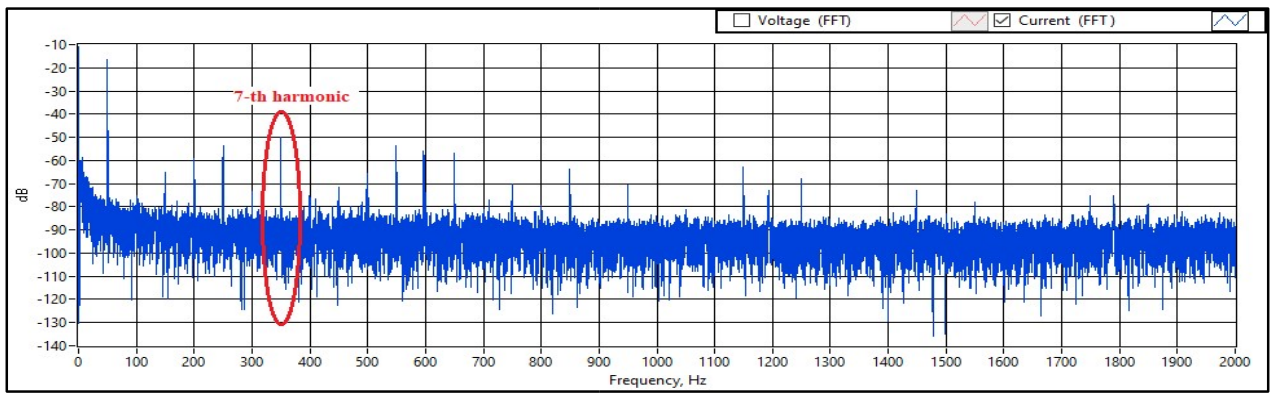

Fig. 14. The spectral analysis of the WPG current, synchronized with the grid voltage.

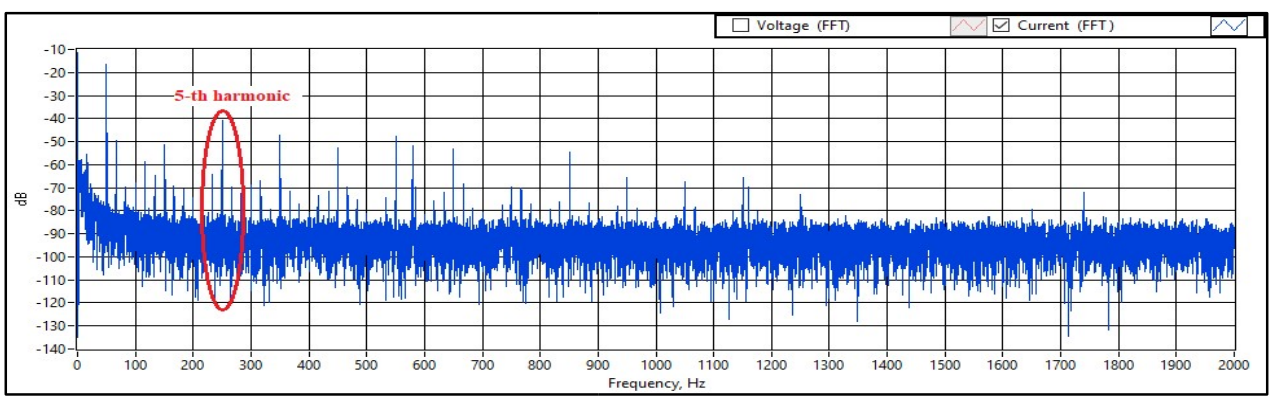

Fig. 15. The spectral analysis of the PVG current, synchronized with the grid voltage.

\section{Conclusions}

The experimental results of the physical RES model, leads to some general conclusions, which can affect the next scientific researches.

- The constructed physical model of the RES system and the simulation of the different power units, represent in an adequate way the processes in the real EPS; 
- The synchronization period and disconnecting times are clearly distinguishable and can be measured despite of the type or model of the used inverter or synchronous generator.

- The spectral analysis of the signals measured by this DAQ measurement set, shows the distribution of the frequency components in a real time, generated from the RES power units in the energy transmission line and EPS;

- By this model and software, can be assessed the influence of the predominant RES part of the energy in the EPS;

- The method for monitoring with DAQ system could be applied over asymmetric loaded three-phase system by involving more measurement channels of the DAQ system.

Along with undisputed utilities of the studied physical model, some disadvantages can be mentioned, which can be a base for future work.

- The switching of the load, using IGBT power switches inputs a lots of higher frequency harmonics, which have to be filtered;

- It will be better, the source DC voltage for simulation of the WPG and PVG to be executed with stabilization, filtering and more flexible way for regulation.

- The validation of the constructed physical model by the present measurements could be useful for studying different types of RES inverters furtherly included in the model.

\section{References}

1. L. Meegahapola, A. Sguarezi, J.S. Bryant, M. Gu, E.R. Conde, R.B.A. Cunha, Energies 2020, 13, 3441, doi.org/10.3390/en13133441

2. G.V.B. Kumar, R.K. Sarojini, K. Palanisamy, S. Padmanaban, J.B. Holm-Nielsen, Energies 2019, 12, 1996, doi.org/10.3390/en12101996

3. T. Markvart, L. Castaner, Practical Handbook of Photovoltaics: Fundamentals \& Aplications, (2003)

4. L. G. Acuña, R.V. Padilla, A. Santander-Mercado, Renewable Energy, 106, 68-77, (2017)

5. L. Gang, Renewable and Sustainable Energy Reviews, 31, 611-621, (2014)

6. Y. Rangelov, N. Nikolaev, Y. Kamenov, K. Gerasimov, IITI 2017, 680, 396-402, (2018), doi:10.1007/978-3-319-68324-9_43

7. P. Yankov, P. Popov, N. Nikolaev, Y. Rangelov, SIELA 2018, (2018), doi:10.1109/SIELA.2018.8447075

8. R. Stanev, BulEF 2020, (2020), doi:10.1109/BulEF51036.2020.9326034

9. J.G. Webster, Measurement, Instrumentation, and Sensors Handbook, (2014)

10. http://www.heyiele.com/Catalogue/Flexible_Rogowski_Coil_With_G1_Integrator633.html

11. I. Nedelchev, H. Zhivomirov, Y. Rangelov, N. Nikolaev, R. Vasilev, E3S Web of Conferences 180, 485-490, (2018), doi.org/10.1051/e3sconf/202018002006 